\title{
Cyclic Vomiting Syndrome in Children: A Review of the Literature
}

\author{
Namir Al-Ansari* \\ Department of Pediatric Gastroenterology, Detroit Medical Center, USA
}

*Corresponding author: Namir Al-Ansari, Department of Pediatric Gastroenterology, Detroit Medical Center, USA.

Received Date: March 30, 2020

Published Date: April 13, 2020

\section{Review}

Cyclic vomiting syndrome (CVS) was first described by Samuel Gee in 1882 [1]. It is classified as a functional gastrointestinal disorder. It is still poorly understood. CVS is a disorder characterized by recurrent, discrete, self-limited stereotypical episodes of nausea and vomiting interspersed with symptom-free intervals or baseline health. It is defined by symptom-based criteria and the absence of positive laboratory, radiographic, and endoscopic testing. During these nausea and vomiting episodes, patients are usually lethargic, pale, have abdominal pain, and anorexia. Episodes are frequently triggered by psychological and physical stress. An association of CVS with migraine headache and abdominal migraine has been described [2,3]. Migraine may have similar symptoms to CVS; both may coexist in same patient, and has high family prevalence in patients with CVS. Affected children are more often girls than boys (60:40). It affects up to $1.9 \%$ of children between $5-15$ years of age [2]. CVS is a source of substantial morbidity: $50 \%$ of children with CVS require intravenous rehydration. Children with CVS miss a mean of 20 days of school each year. The average cost of emergency department visits, hospital stays, diagnostic tests, and missed work is estimated at more than $\$ 17,000$ yearly per child with CVS [4]. The NASPGHAN and Rome IV diagnostic criteria for CVS are [5,6]:

\section{NASPGHAN criteria}

1. At least 5 attacks in any interval, or a minimum of 3 attacks during a 6-mo period.

2. Episodic attacks of intense nausea and vomiting lasting 1 $\mathrm{h}-10$ days and occurring at least $1 \mathrm{wk}$ apart.

3. Stereotypical pattern and symptoms in the individual patient.
4. Vomiting during attacks occurs at least 4 times/h for at least $1 \mathrm{~h}$.

5. Return to baseline health between episodes.

6. Not attributed to another disorder.

\section{Rome IV criteria}

1) Two or more episodes of intense, unremitting nausea and vomiting lasting hours to days within 6-month period.

2) Stereotypical episodes.

3) Episodes separated by weeks to months with interepisodic return to baseline health.

\section{Etiology}

The etiology and pathogenesis of CVS still remains unknown. Hypotheses have been suggested as possible etiologies: migraine variant, mitochondrial diseases including mitochondrial fatty acid oxidation disorders, gastrointestinal motility disorder, autonomic dysfunction, abdominal epilepsy, ion channel dysfunction, and altered psychodynamics [7].

\section{Clinical Features}

CVS is a disorder characterized by recurrent, stereotypic episodes of nausea and vomiting lasting for hours to days with symptom-free intervals lasting weeks to months. CVS typically has 3 phases: prodromal phase, emetic phase, and inter-episodic or asymptomatic phase. Triggering factors for vomiting episodes may be identified in $40-80 \%$ of CVS patients. Common triggering factors are psychological stress, infections, physical exhaustion, and specific foods (like chocolate, cheese). Prodromal symptoms 
may precede vomiting episode in one third of patients. Prodromal symptoms of CVS consist of anxiety, change in mood, headache, dizziness, nausea, abdominal pain, fatigue, and temperature changes [7]. Vomiting episodes of CVS usually begin early in the morning. Patients show intensive emesis and episodes usually continue from 2 hours to 10 days. Continuous nausea and vomiting is usually associated with abdominal pain and retching. The emetic phase is characterized by listlessness, pallor, flushing, diaphoresis, drooling, loose stools, dizziness, photophobia, intolerance to noise, tachycardia, mild elevation of blood pressure, and even low-grade fever or hypothermia. The intense nausea persists even after evacuation of stomach contents and many describe it as the most distressing symptom only relieved by sleep. Due to the unrelenting nausea, patients are debilitated, listless, withdrawn, and generally unable to communicate or walk [6,7]. Complications secondary to intensive prolonged vomiting include dehydration, electrolyte imbalance, gastritis, esophagitis, Mallory-Weiss tear, and weight loss. The emetic phase is followed by the recovery phase which can be very quick and patients are suddenly able to eat and interact.

\section{Diagnosis}

There are no specific laboratory markers for CVS. The diagnosis relies on fulfilling the clinical criteria ofCVSin patients with recurrent episodes of vomiting in the absence of another explanation for the symptoms. Thorough history taking and physical examination are essential to diagnose CVS and presentation helps identify those patients in whom further diagnostic testing is prudent. Suspicious symptoms and physical findings include the following [5]:

a. Bilious vomiting, abdominal tenderness and/or severe abdominal pain.

b. Attacks precipitated by intercurrent illness, fasting, and/ or high protein meal.

c. Abnormalities on neurological examination including severe alteration of mental status, abnormal eye movements, papilledema, motor asymmetry, and/or gait abnormality (ataxia).

d. Progressively worsening episodes or conversion to a continuous or chronic pattern.

\section{Differential Diagnosis}

Differential diagnostic approaches are recommended depending upon the presenting symptoms and signs other than vomiting $[5,6,9]$.

1. Gastrointestinal causes: Intermittent bowel obstruction (malrotation with volvulus, duplication cyst, and postoperative adhesions/stricture), gallbladder disease, pancreatitis, hepatitis, esophagitis, gastritis, eosinophilic esophagitis, and celiac disease. Patients may present with abdominal pain, bilious vomiting and may be hematemesis with recurrent episodes of vomiting. Diagnostic tests may include upper GI series, upper endoscopy, amylase and lipase, liver enzymes, and abdominal ultrasound. Abnormal results warrant further testing.

2. Infectious causes: Chronic sinusitis, otitis media.

3. Genitourinary causes: Uretero-pelvic junction obstruction. Diagnostic tests include imaging tests to evaluate the urinary tract.

4. Metabolic causes: Mitochondrial disorders, fatty acid oxidation defects, urea cycle defects, disorders of organic and amino acid metabolism. These conditions should be considered when vomiting episodes are precipitated by fasting, high-protein meals, or intermittent illness. Diagnosis of these disorders is established with metabolic testing including plasma amino acid and urine organic acid analysis.

5. Endocrine causes: Addison's disease, diabetic ketoacidosis, pheochromocytoma.

6. Neurological causes: Migraine, seizures, posterior fossa or hypothalamic tumor, Chiari malformation, hydrocephalus, subdural hematoma. These conditions are considered in patients presenting with abnormal neurological findings such as altered mental status, ataxia, seizures, papilledema, or abnormal eye movements. Patients should have neurological evaluation and brain imaging such as MRI or CT scan.

7. psychiatric causes: Bulimia nervosa, Munchausen by proxy. Cyclic vomiting has been described in association with chronic cannabis use described as cannabis hyperemesis syndrome [8]. A number of comorbidities are identified in association with CVS. The most common include anxiety and depression, irritable bowel syndrome, limited stamina or chronic fatigue, sleep disturbance, postural tachycardia syndrome, and complex regional pain syndrome [6].

\section{Treatment}

Management of CVS is challenging. The clinical course of CVS includes the episodic phase with its prodromal and vomiting course and the symptom-free phase. Each phase has its therapeutic implications $[5-7,10]$. Treatment can be divided into:

\section{Prophylactic/preventative therapy}

The goal is to prevent subsequent episodes. Life style modifications may be recommended first. Prophylaxis is focused on identification and avoidance of known potential triggering factors of vomiting episodes. Management strategies including avoidance of stressful events, excessive excitement, sleep deprivation, energydepleted states (fasting, acute infection, and acute illness), and triggering foods (e.g., chocolate, cheese). The use of supplemental carbohydrate to provide additional energy during times of high energy demands. Close screening for psychiatric comorbidity is essential. Prophylactic therapy is warranted for patients with more frequent or severe episodes. Prophylactic medications used include amitriptyline, cyproheptadine, propranolol, and phenobarbital. Amitriptyline $(0.2-0.3 \mathrm{mg} / \mathrm{kg} /$ day; titrate to $1-1.5 \mathrm{mg} / \mathrm{kg} /$ day 
q.h.s), or propranolol (2-3mg/kg/day q.h.s) are recommended for children older than 5 years. Side effects of amitriptyline (a tricyclic antidepressant) include sedation, dry mouth, constipation, chronic fatigue, mood changes, and QTc prolongation. Propranolol side effects hypotension, bradycardia, fatigue, and depression. Cyproheptadine or propranolol are recommended for children 5 years or younger. Cyproheptadine (antihistamine and serotonin receptor antagonist) side effects include sedation, weight gain, and anticholinergic effect. Other medication used is phenobarbital (2-3mg/kg/day q.h.s). Mitochondrial supplements are used as adjunctive prophylactic therapy. Those include L-carnitine (50$100 \mathrm{mg} / \mathrm{kg} /$ day divided b.i.d), Co-enzyme Q10 (10mg/kg/day divided b.i.d $\leq 600 \mathrm{mg} /$ day $)$, and riboflavin $(10 \mathrm{mg} / \mathrm{kg} /$ day divided b.i.d $\leq 400 \mathrm{mg} /$ day).

\section{Abortive therapy}

The goal of the abortive therapy is to intervene during the prodromal phase at the beginning of the vomiting episode in the hope of terminating the attack. Abortive therapy is usually considered for patients who have sporadic episodes and/or prefer not taking prophylaxis for those who have break-through episodes while on prophylaxis. Therapies during prodromal phase include control of stressful lifestyle (lying down in a dark and quiet environment), high carbohydrate ingestion, and use of medications. Recommended medications include (anti-emetic agents (ondansetron and granisetron), anti-anxiety agents (lorazepam), and anti-migraine agents (sumatriptan and zolmitriptan). The effectiveness of these therapies was not approved due to the lack of data.

\section{Supportive therapy}

Supportive or rescue care is used when the vomiting becomes well-established and an episode fails to respond to any abortive strategies. The goal of therapy during emetic phase is termination of vomiting episode and prevention of complications such as dehydration and electrolyte imbalance. Treatment is focused on providing relief from nausea, vomiting, and abdominal pain. A nonstimulating environment including quiet and dark room may be helpful. Intravenous fluids are used for correction of dehydration and electrolyte imbalance. Antiemetic therapy, analgesics, and sedation are used for relief from unrelenting nausea, vomiting, and abdominal pain. Gastric acid suppressing agents may be helpful.
Ondansetron has been widely used antiemetic. Diphenhydramine, lorazepam, or chlorpromazine combined with diphenhydramine used to induce sedation because sedation is often the only means of providing good relief from the unrelenting nausea and abdominal pain. Supportive care should be continued until the patient is able to resume oral intake.

\section{Long-term outcome of CVS}

It is not easy to predict clinical course and long-term outcome of CVS in children due to limited data (7). Many patients eventually recover from CVS, but some patients continue to have CVS or suffer from migraine headache later.

\section{Acknowledgement}

None.

\section{Conflict of Interest}

No conflict of interest.

\section{References}

1. Gee S (1882) On fitful or recurrent vomiting.St. Bart Hosp Rev 18: 1-6.

2. Abu-Arafeh I, Russel G (1995) Cyclic vomiting syndrome in children: a population based study. J Pediatr Gastroenterol Nutr 21: 454-458.

3. Stickler GB (2005) Relationship between cyclic vomiting syndrome and migraine. Clan Pediatric (Phyla) 44: 505-508.

4. Li BU, Baling J (2000) Cyclic vomiting syndrome: evolution in our understanding of a brain-gut disorder. Adv. Pediatric 47: 117-160.

5. Li BU, Lefevre F, Chelimsky GG, Boles RG, Nelson SP, et al. (2008) North American society for pediatric gastroenterology, hepatology and nutrition consensus statement on the diagnosis and management of cyclic vomiting syndrome. J Pediatr Gastroenterol Nutr 47: 379-393.

6. Kovacic K, Sood M, Venkatesan T (2018) Cyclic Vomiting Syndrome in Children and Adults: What is new in 2018. Curr Gastroenterol Rep 20(10): 46.

7. Yang HR (2010) Recent concepts on cyclic vomiting syndrome in children. J Neurigastroenterol Motil 16(2): 139-147.

8. Allen JH, de Moore GM, Heddle R, Twartz JC (2004) Cannabinoid hyperemesis: cyclical hyperemesis in association with chronic cannabis abuse. Gut 53: 1566-1570.

9. Trandafir LM, Burlacu M, Tudose AAM, Nascu I, Grigorescu-Sido P (2016) Problems in the Diagnosis of Cyclic Vomiting Syndrome in Children. Maedica (Buchar) 11(2): 150-153.

10. Fleisher D (1995) Management of cyclic vomiting syndrome. J Pediatr Gastroenterol Nutr 21(Suppl. 1): S52-S56. 